\title{
Controlled Actuation of Liquid Marbles on a Dielectric
}

\section{Xiangyu Fu, Yage Zhang, Hao Yuan, Bernard P. Binks, and Ho Cheung Shum}

This document is the Accepted Manuscript version of a Published Work that appeared in final form in ACS Applied Materials \& Interfaces, copyright (C) American Chemical Society after peer review and technical editing by the publisher. To access the final edited and published work see https://pubs.acs.org/doi/full/10.1021/acsami.8b13111

ABSTRACT: Motivated by the great potential of droplet microreactors for chemical and biological applications, a general and robust method utilizing an electric field is developed for sustained, directional and two-dimensional manipulation of nonwetting droplets (termed "liquid marbles"). With the understanding of the mechanism of actuation, this method allows individual liquid marbles to be actuated and coalesced on demand by fine-tuning the driving voltage. Moreover, in our system, crosscontamination between marbles during manipulation is avoided as confirmed by the absence of any trace DNA after amplification using a loop-mediated isothermal amplification reaction.

The manipulation of liquid droplets is revolutionizing many liquid-based applications in biological detection and clinical diagnosis.(1-3) However, the biological molecules in liquid droplets can adsorb in a nonspecific manner to solid surfaces during manipulation.(4) The resultant contamination can significantly impede the manipulation of droplets, and even render the droplets useless for further biological analysis.(5) In particular, the adsorption of only a minute amount of DNA and RNA can lead to severe cross-contamination, as the contaminants could be amplified in subsequent steps, such as in polymerase chain reaction (PCR) and loop-mediated isothermal amplification (LAMP) resulting in erroneous results. To overcome these limitations, researchers can achieve nonwetting droplets by coating the interfaces of water droplets with hydrophobic particles. The resulting droplets, often known as "liquid marbles",(6-8) have demonstrated great potential as microreactors for material fabrication,(9) cell culture applications,(10) and chemical reactions.(11-13) Nevertheless, manual movement and merging of liquid marbles can become inefficient and tedious when a large quantity of samples are involved. Therefore, controlled and sustained manipulation of liquid marbles automated by external stimulation is highly desired.

To date, various methods have been proposed to transport liquid marbles. Typical examples(14) include the use of magnetic $(15,16)$ or photosensitive $(17,18)$ particles to form liquid marbles with triggered motion. However, these methods are applicable only to liquid marbles that can be stabilized by the specific types of particles. Another method is to utilize surface flow $(19,20)$ or air flow(21) to actuate floating liquid marbles. However, it is difficult to generate a sufficiently large force using surface flow to break the particle shell and coalesce two liquid marbles for initiating a reaction or detection. Therefore, a robust method, which does not pose specific requirements of the marbles involved, to actuate liquid marbles is yet to be developed.

An electric field has shown great potential in actuating liquid marbles in a tunable and contactless manner, as confirmed by the deformation of the liquid marble interface with electrodes inserted into the marbles.(22-24) When placed between two parallel plate electrodes,(25) a liquid marble on 
a superhydrophobic surface is observed to move. However, the resultant movement of the liquid marble, although successfully proven conceptually, cannot offer a deep understanding of how the electric field can actuate a liquid marble to move precisely. This may be due to the lack of a systematic design that triggers controlled movement for liquid marbles. In this work, we present a general and robust method for sustained, directional and two-dimensional manipulation of liquid marbles. To actuate the motion and induce the coalescence of individual liquid marbles, our method incorporates an inkjet-printed electric circuit covered by a dielectric layer and DC voltages applied to neighboring electrodes. We have studied the critical voltage to actuate the liquid marbles, and shown that the amount of electrostatic energy released to accelerate the marble is positiondependent.

We have developed a compact and versatile system for controlled manipulation of liquid marbles by incorporating a device that comprises three key components: a substrate, an open array of electrodes and a layer of dielectric insulator, as illustrated schematically in Figure 1a. Electric circuits as thin as $\sim 1 \mu \mathrm{m}$ are fabricated by an inkjet printer,(26) then coated with $\sim 10 \mu \mathrm{m}$ polydimethylsiloxane (PDMS) as the dielectric layer (see experimental details in the Supporting Information). A liquid marble is formed by coating a liquid droplet with hydrophobic silica nanoparticles (particle/inner liquid ratio $\sim 0.1 \mathrm{mg} / \mathrm{mL}$ ) and then placed on one of the electrodes in the device, covering a part of a neighboring electrode as well. The electrode covered by the liquid marble is grounded while a positive direct current (DC) voltage is preset and applied to the neighboring electrode. At zero or low preset voltage, the liquid marble remains stationary on the device. When the preset value of the applied voltage increases and approaches a critical value VC, the liquid marble starts to move toward the charged electrode by rolling, which is captured by a high-speed camera as shown in Figure $1 \mathrm{~b}$ and indicated by the nonzero velocity in Figure $1 \mathrm{c}$. We define the value of this minimum driving voltage that can actuate a liquid marble to move as the critical actuation voltage for the liquid marbles. For example, at the critical voltage of $780 \mathrm{~V}$, a water marble is actuated and reaches a maximum velocity of $7.4 \mathrm{~mm} / \mathrm{s}$ (see velocity profile in Figure S5).

\section{[FIG 1]}

To actuate a pure droplet on a dielectric, the well-studied electrowetting phenomenon leads to asymmetric contact angles across the droplet resulting in droplet motion.(4) However, for liquid marbles, the mismatch between advancing and receding contact angles is as small as $\sim 1.1^{\circ}$ (see Figure S1). Because the change in the contact angle is an observable effect instead of a requirement for actuating liquid marbles, the actuation of liquid marbles should depend on the electrostatic polarization of the inner liquid in response to the electric field. Therefore, the electrical conductivity $\kappa$ and the relative electrical permittivity $\varepsilon$ of the inner liquid is expected to affect the critical voltage VC. Indeed, VC decreases as $\mathrm{K}$ increases by adding different concentrations of $\mathrm{NaCl}$ ranging from 0.001 to $1 \mathrm{M}$ to deionized (DI) water, as shown in Figure 2a. Similarly, VC decreases as $\varepsilon$ increases, as shown in Figure $2 \mathrm{~b}$. We attribute these results to the ability of the liquid contents to provide a larger number of ions at the liquid-air interface. Hence, given the same applied voltage, a larger electric stress is exerted on the liquid marble. These results imply that the driving force for the actuation comes from the electrostatic polarization of the inner liquid.

[FIG 2]

Interestingly, the critical actuation voltage VC changes and depends crucially on the initial position of the liquid marble relative to the electrodes before actuation. The different initial positions can be 
represented by normalizing the distance between the marble centroid and the symmetry line of the pairing electrodes $d$ with respect to the marble radius $R$, as shown in Figure $2 c$. A larger $d / R$ ratio indicates that the marble is farther away from the charged electrode. With a larger $d / R$ ratio ranging from 0.1 to 1.0, the critical actuation voltage VC becomes larger. Such a correlation also holds true for different liquid volumes (see Figure S2).

To understand the position-dependent critical actuation voltage, we investigate further and analyze the changes taking place at the boundary of the polarized interfacial area, as illustrated schematically in Figure $2 \mathrm{~d}$. In response to the surrounding electric field, the interface of the liquid marble is electrostatically polarized and carries a surface charge q. Specifically, the contact area between a liquid marble and the device is where charges build up. Before actuation, since no electric potential difference exists for the ground electrode area, the polarized interfacial area of a liquid marble can be defined as SO and measured directly from the top-view. During actuation, the polarized interfacial area increases and reaches a maximum area $\mathrm{Sr}$, which equals the contact area between the marble and the device, where is the capillary length and $\rho, \gamma$ are the density and surface tension of the liquid, respectively. $(10,11)$ Therefore, the total increase in the polarized interfacial area is $\Delta \mathrm{S}=\mathrm{Sr}-\mathrm{SO}$.

Considering that the liquid marble moves by rolling instead of sliding, the role of the induced electric stress fe is key to understanding the actuation mechanism. Because of electrostatic polarization, an electric stress fe is induced at the interface by the local electric field $E$, which scales as.$(24,25)$ For a small displacement $\Delta x$ of the liquid marble, fe pulls the interface by compressing and pinning the interface to the substrate over a height $\Delta y$, as shown in Figure $2 \mathrm{~d}$. The work done per unit area by fe at the interface is thus. The local electric field strength $E$ in the $y$ direction can be estimated as, where $V$ is the applied electric voltage,,$\lambda$ is the geometric factor to calculate the $y$-direction component given by $1 / \tan -1(\mathrm{dD} / \mathrm{dgap}) 2$, dgap is the size of the gap between two electrodes and $\mathrm{dD}$ is the thickness of the dielectric layer (see Figures S6 and S7 for the influence of particles).(27) Notably, the work done by fe is also the electrostatic energy of the system released to accelerate the liquid marble. fe releases maximum electrostatic energy when the polarized interfacial area $S$ reaches a maximum, since any further increase in polarized interfacial charges on one side of the marble causes the discharging of the same amount on the opposite side. As a result, the liquid marble can no longer experience a net accelerating electric stress exerting at the interface. We can now calculate the released electrostatic energy at this instant, as determined by the electric stress exerting on the interface, the distance between the interface and the electrode and the change in the charged interfacial area:(28,29)

where $V$ is the critical voltage, $\varepsilon 0=8.85 \times 10-12 \mathrm{~F} / \mathrm{m}$ is the permittivity of vacuum, and $\varepsilon r=80.1$ is the relative permittivity of water.

According to our interpretation, at this instant the liquid marble should reach its peak velocity of the entire movement. We can approximate the kinetic energy of the marble at peak velocity as where $\mathrm{m}$ is the mass of the marble and $\mathrm{v}$ is the peak velocity. The energy conversion between released electrostatic energy and kinetic energy, Ue $\sim$ Uk, gives 
(2)

This represents our predicted scaling relationship that is used to analyze the position-dependent critical voltage for liquid marbles of different volumes. The corresponding peak velocity $v$ for all groups of liquid marbles is also measured (see Figure S3). The scaling between the kinetic energy and the electrostatic energy falls around a line with a slope of unity, as seen in Figure 2e. The experimental data indeed agree with our prediction and confirm our interpretation that maximum electrostatic energy is released when the contact area between the marble and the substrate is fully polarized.

More importantly, with the calculated electrostatic energy, we can now estimate the driving force to actuate liquid marbles. The average driving force during acceleration varies with the distance $d$ normalized by the capillary length $\mathrm{k}-1$ in Figure $2 \mathrm{f}$. The average driving force for a $4 \mu \mathrm{L}$ marble is around $0.1 \mu \mathrm{N}$. Meanwhile, a larger liquid marble requires a larger driving force to actuate. For a marble with a volume of $8 \mu \mathrm{L}$, the driving force increases to over $0.3 \mu \mathrm{N}$. For marbles of the same volume, when the marble is farther away from the charged electrode, the required driving force is larger. Therefore, our findings allow us to precisely control the driving force to actuate liquid marbles.

Our understanding of the mechanism of actuation allows controlled motion through manipulation of the relevant driving forces. However, whether our system is reliable for sustained and continuous actuation of liquid marbles is yet to be examined. To realize a sustained and continuous actuation, an electric voltage needs to be sequentially applied to a series of electrodes. Regardless of the initial position, a liquid marble should be actuated and then stopped at the charged electrode before it is ready for the subsequent actuation. Before actuation, the distance between the centroid of the marbles and the symmetry line of the charged electrode is measured. After actuation, the total displacement of the marble is measured. The results show that the desired and actual moving distance of the marble stay roughly the same, as shown in Figure 3a, indicating that regardless of their size, the liquid marbles stop at the charged electrode after actuation.

[FIG 3]

To further examine the reliability of our system, we placed two liquid marbles dyed yellow and green far from each other for actuation. An electric potential is sequentially applied to a series of electrodes. First, the yellow marble is actuated to move horizontally, as seen in Figure $3 \mathrm{~b}$ (for full movie, see Video S1). The green marble is then actuated by a diagonal pair of electrodes, moving in a diagonal direction. Lastly, a potential is applied to the neighboring marbles causing them to coalesce into one.

Moreover, we confirm the avoidance of cross-contamination between liquid marbles by demonstrating the absence of any trace DNA after a LAMP reaction. Specifically, we actuate a liquid marble containing $1 \times 104$ copies/ $\mu \mathrm{L}$ target DNA to move back-and-forth along a predefined path for 100 times, as shown in Figure 4a. Then another liquid marble containing DI water is actuated backand-forth along the same path for 100 times (surface coverage of particles is shown in Figures $\mathrm{S} 8$ and S9). Afterward, the liquid in the second liquid marble is extracted and mixed with LAMP buffers and primer of the target DNA separately, because any amplification by the LAMP reaction would 
indicate the extent of cross-contamination. The resultant mixture is then heated at $63^{\circ} \mathrm{C}$ for $1 \mathrm{~h}$ in the Real Time PCR machine. A flat amplification curve is acquired after heating, indicating that no amplification proceeded as shown in Figure $4 b$ (control groups are shown in Figure S4). The results suggest that no detectable DNA is contained inside the second liquid marble, confirming that no cross-contamination between the two marbles occurs.

[FIG 4]

To investigate the potential cross-contamination when the particle shell in one of the marbles is partially damaged, we repeat the above-mentioned procedures. Specifically, we drop a liquid marble from a height of $\sim 3.5 \mathrm{~cm}$ on a PDMS substrate to partly damage the particle shell (the surface coverage of the particles is shown in Figure S10). We damage the particle shell of either the first DNA marble or the second water marble, and obtain flat amplification curves (Figure $4 \mathrm{~b}$ ) for both groups, indicating that no DNA is detected in the second water marble. However, when the particle shell of both marbles is damaged, an S-shaped curve is found in the LAMP reaction (Figure 4b), indicating cross-contamination. Therefore, at least one well-coated liquid marble is required to prevent the cross-contamination between two liquid marbles when carrying out biochemical reactions on the proposed platform. We believe that the reliability and versatility of our system will extend the range of integrated processes and applications that can be carried out in liquid marbles.

In summary, we have developed a general and robust method for controlled manipulation of liquid marbles. By utilizing an electric field on inkjet-printed devices, we have systematically studied the controlled actuation of liquid marbles on a dielectric. Using our platform, a liquid marble can be actuated to move when the applied voltage to the electrode is above a critical value. Interestingly, the critical actuation voltage is highly dependent on the initial positions of the liquid marble relative to the electrodes. We attribute this finding to the driving forces that vary with the electrostatically polarized interfacial area; hence, the amount of electrostatic energy released to accelerate the marble to reach peak velocity is position-dependent, as confirmed experimentally. These results allow us to estimate the driving force as well as the displacements of actuation. By fine-tuning the driving voltage, our system can actuate individual liquid marbles and coalesce them. Moreover, by demonstrating the absence of any trace DNA after amplification using a LAMP reaction, our system is confirmed to avoid the cross-contamination between liquid marbles. The controlled manipulation and avoidance of cross-contamination achieved using our platform will enable future biological applications of liquid marbles.

\section{REFERENCES}

(1) Whitesides, G. M. The Origins and the Future of Microfluidics. Nature 2006, 442, 368-373.

(2) Wang, H.; Gu, H.; Chen, Z.; Shang, L.; Zhao, Z.; Gu, Z.; Zhao, Y. Enzymatic Inverse Opal Hydrogel Particles for Biocatalyst. ACS Appl. Mater. Interfaces 2017, 9, 12914-12918.

(3) Song, Y.; Michaels, T. C. T.; Ma, Q.; Liu, Z.; Yuan, H.; Takayama, S.; Knowles, T. P. J.; Shum, H. C. Budding-like Division of All-Aqueous Emulsion Droplets Modulated by Networks of Protein Nanofibrils. Nat. Commun. 2018, 9, 2110. 
(4) Link, D. R.; Grasland-Mongrain, E.; Duri, A.; Sarrazin, F.; Cheng, Z.; Cristobal, G.; Marquez, M.; Weitz, D. A. Electric Control of Droplets in Microfluidic Devices. Angew. Chem., Int. Ed. 2006, 45, 2556-2560.

(5) Choi, K.; Ng, A. H. C.; Fobel, R.; Wheeler, A. R. Digital Microfluidics. Annu. Rev. Anal. Chem. 2012, $5,413-440$.

(6) Aussillous, P.; Queré , D. Liquid Marbles.' Nature 2001, 411, 924-927.

(7) Liu, Z.; Fu, X.; Binks, B. P.; Shum, H. C. Mechanical Compression to Characterize the Robustness of Liquid Marbles. Langmuir 2015, 31, 11236-11242.

(8) McHale, G.; Newton, M. I. Liquid Marbles: Topical Context within Soft Matter and Recent Progress. Soft Matter 2015, 11, 2530-2546.

(9) Chu, Y.; Wang, Z.; Pan, Q. Constructing Robust Liquid Marbles for Miniaturized Synthesis of Graphene/Ag Nanocomposite. ACS Appl. Mater. Interfaces 2014, 6, 8378-8386.

(10) Wang, D.; Zhu, L.; Chen, J. F.; Dai, L. Liquid Marbles Based on Magnetic Upconversion Nanoparticles as Magnetically and Optically Responsive Miniature Reactors for Photocatalysis and Photodynamic Therapy. Angew. Chem., Int. Ed. 2016, 55, 10795-10799.

(11) Dupin, D.; Armes, S. P.; Fujii, S. Stimulus-Responsive Liquid Marbles. J. Am. Chem. Soc. 2009, 131, 5386-5387.

(12) Fujii, S.; Suzaki, M.; Armes, S. P.; Dupin, D.; Hamasaki, S.; Aono, K.; Nakamura, Y. Liquid Marbles Prepared from pH-Responsive Sterically Stabilized Latex Particles. Langmuir 2011, 27, 8067-8074.

(13) Zhao, Y.; Gu, H.; Xie, Z.; Shum, H. C.; Wang, B.; Gu, Z. Bioinspired Multifunctional Janus Particles for Droplet Manipulation. J. Am. Chem. Soc. 2013, 135, 54-57.

(14) Fujii, S.; Yusa, S.; Nakamura, Y. Stimuli-Responsive Liquid Marbles: Controlling Structure, Shape, Stability, and Motion. Adv. Funct. Mater. 2016, 26, 7206-7223.

(15) Xue, Y.; Wang, H.; Zhao, Y.; Dai, L.; Feng, L.; Wang, X.; Lin, T. Magnetic Liquid Marbles: A "Precise" Miniature Reactor. Adv. Mater. 2010, 22, 4814-4818.

(16) Zhang, L.; Cha, D.; Wang, P. Remotely Controllable Liquid Marbles. Adv. Mater. 2012, 24, 4756-4760.

(17) Chu, Y.; Liu, F.; Qin, L.; Pan, Q. Remote Manipulation of a Microdroplet in Water by NearInfrared Laser. ACS Appl. Mater. Interfaces 2016, 8, 1273-1279.

(18) Paven, M.; Mayama, H.; Sekido, T.; Butt, H.-J.; Nakamura, Y.; Fujii, S. Light-Driven Delivery and Release of Materials Using Liquid Marbles. Adv. Funct. Mater. 2016, 26, 3199-3206.

(19) Bormashenko, E.; Bormashenko, Y.; Grynyov, R.; Aharoni, H.; Whyman, G.; Binks, B. P. SelfPropulsion of Liquid Marbles: Leidenfrost-like Levitation Driven by Marangoni Flow. J. Phys. Chem. C 2015, 119, 9910-9915.

(20) Kavokine, N.; Anyfantakis, M.; Morel, M.; Rudiuk, S.; Bickel, T.; Baigl, D. Light-Driven Transport of a Liquid Marble with and against Surface Flows. Angew. Chem., Int. Ed. 2016, 55, 11183-11187. 
(21) Kawashima, H.; Paven, M.; Mayama, H.; Butt, H. J.; Nakamura, Y.; Fujii, S. Transfer of Materials from Water to Solid Surfaces Using Liquid Marbles. ACS Appl. Mater. Interfaces 2017, 9, 33351-33359.

(22) McHale, G.; Herbertson, D. L.; Elliott, S. J.; Shirtcliffe, N. J.; Newton, M. I. Electrowetting of Nonwetting Liquids and Liquid Marbles. Langmuir 2007, 23, 918-924.

(23) Bormashenko, E.; Pogreb, R.; Stein, T.; Whyman, G.; Schiffer, M.; Aurbach, D. Electrically Deformable Liquid Marbles. J. Adhes. Sci. Technol. 2011, 25, 1371-1377.

(24) Liu, Z.; Fu, X.; Binks, B. P.; Shum, H. C. Coalescence of Electrically Charged Liquid Marbles. Soft Matter 2017, 13, 119-124.

(25) Bormashenko, E.; Pogreb, R.; Balter, R.; Gendelman, O.; Aurbach, D. Composite Non-Stick Droplets and Their Actuation with Electric Field. Appl. Phys. Lett. 2012, 100, 151601.

(26) Dixon, C.; Ng, A. H. C.; Fobel, R.; Miltenburg, M. B.; Wheeler, A. R. An Inkjet Printed, Roll-Coated Digital Microfluidic Device for Inexpensive, Miniaturized Diagnostic Assays. Lab Chip 2016, 16, 4560-4568.

(27) Abdelgawad, M.; Park, P.; Wheeler, A. R. Optimization of Device Geometry in Single-Plate Digital Microfluidics. J. Appl. Phys. 2009, 105, No. 094506.

(28) Jones, T. B. On the Relationship of Dielectrophoresis and Electrowetting. Langmuir 2002, 18, 4437-4443.

(29) Chatterjee, D.; Shepherd, H.; Garrell, R. L. Electromechanical Model for Actuating Liquids in a Two-Plate Droplet Microfluidic Device. Lab Chip 2009, 9, 1219-1229.

Figures

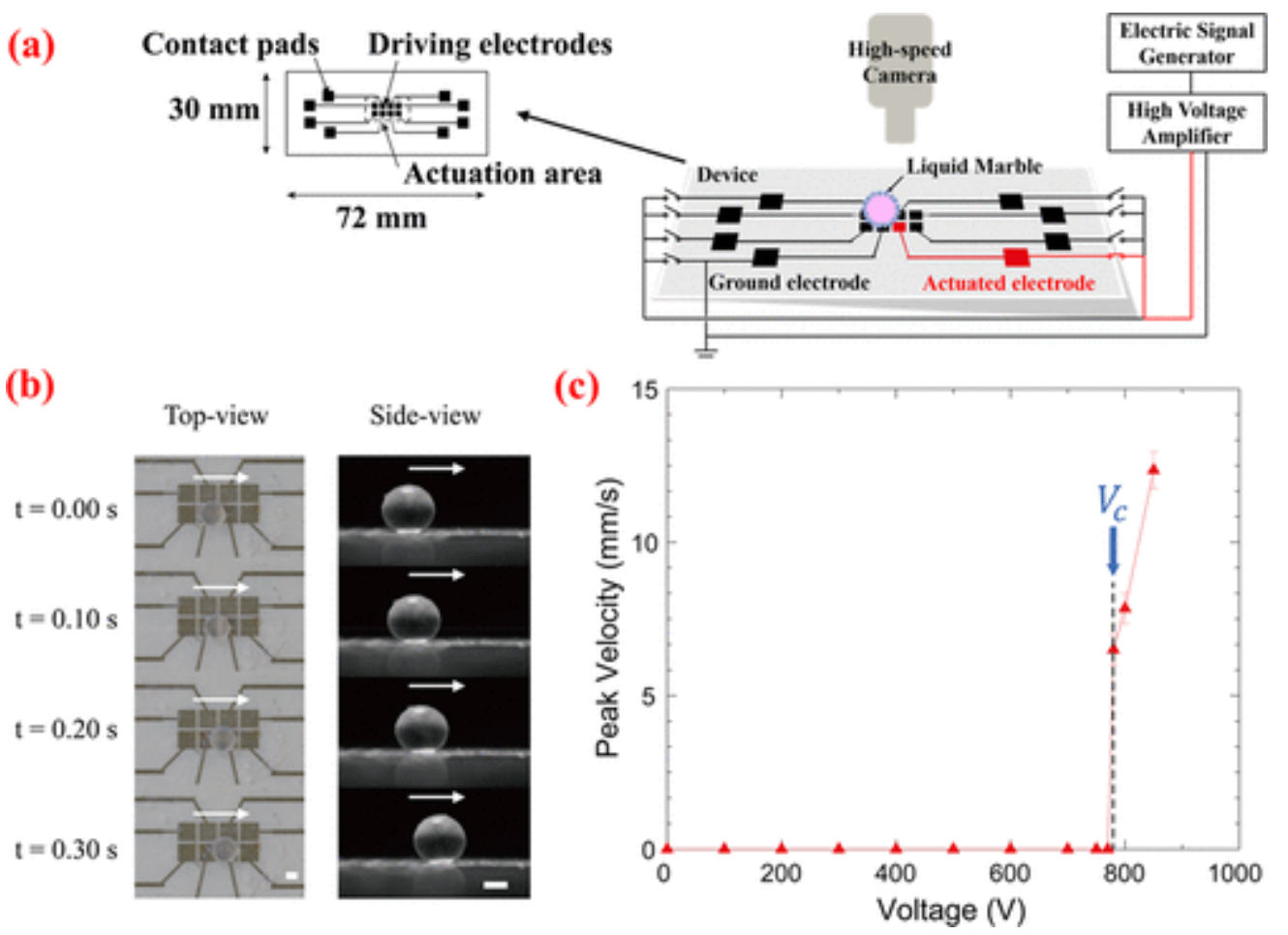


Figure 1. (a) Schematic of the configuration of the custom-built system for the actuation of liquid marbles. The area in black color in the top-view schematic of the device is the designed circuit for printing the conductive silver ink. An open array of electrodes and contact pads for interfacing with the power and control system are printed onto the printing media. The electric potential $V$ is applied through the electrode of the bottom plate in red color. A signal generator is used to output a DC voltage to an amplifier with a magnification of 1000 times. The corresponding electrode of the device is then connected to the output of the amplifier. The movement of the liquid marble is monitored by a high-speed camera. (b) Photographs showing that a liquid marble deposited on the device moves toward the charged electrode, pictured from both the top and side. The liquid marble is a water droplet of volume $5 \mu \mathrm{L}$ coated with silica nanoparticles. Scale bar $=1 \mathrm{~mm}$. (c) Evolution of the peak velocity of the movement of the liquid marble when increasing the preset voltage applied to the electrodes. Here, the liquid marbles are formed by coating water droplets of volume $5 \mu \mathrm{L}$ with silica nanoparticles.

Figure 2

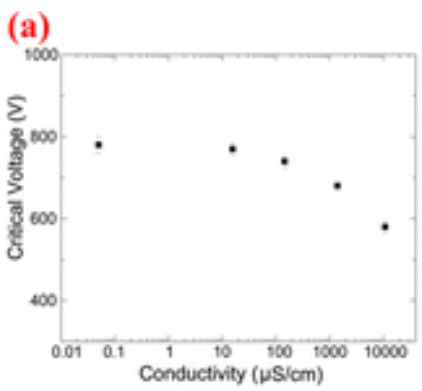

(b)

(c)
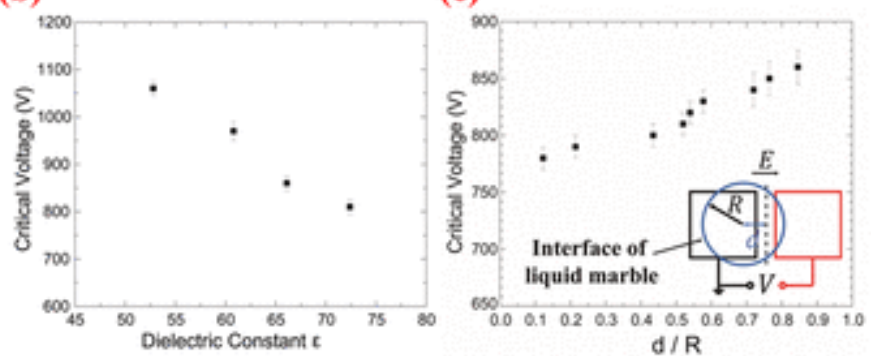

(d)

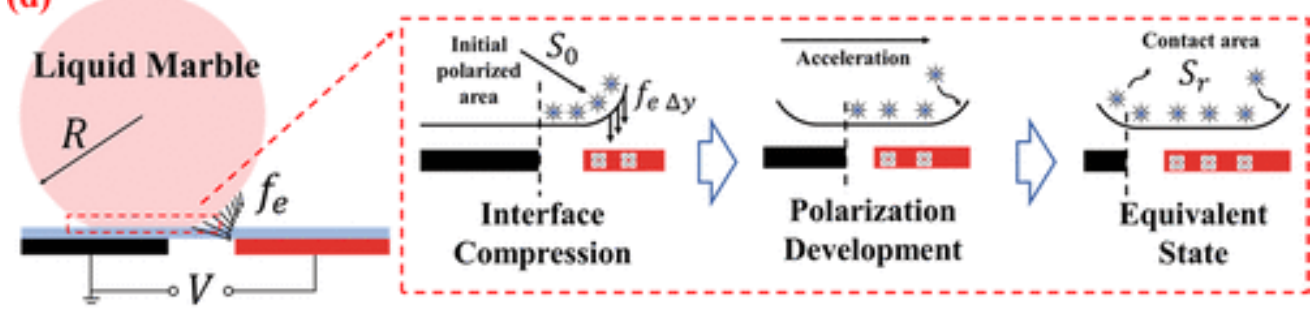

(e)

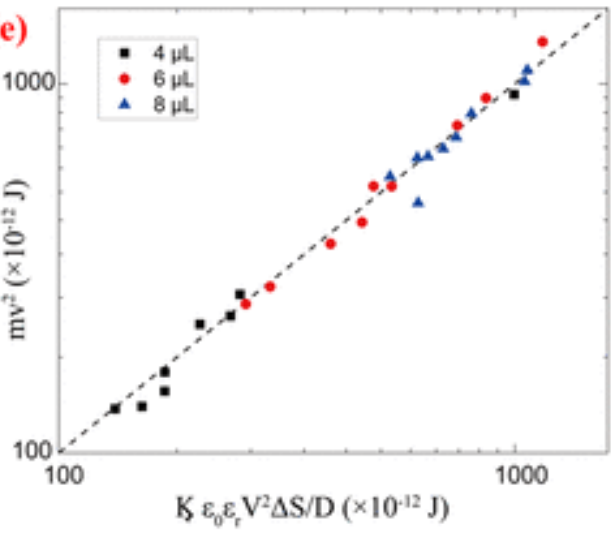

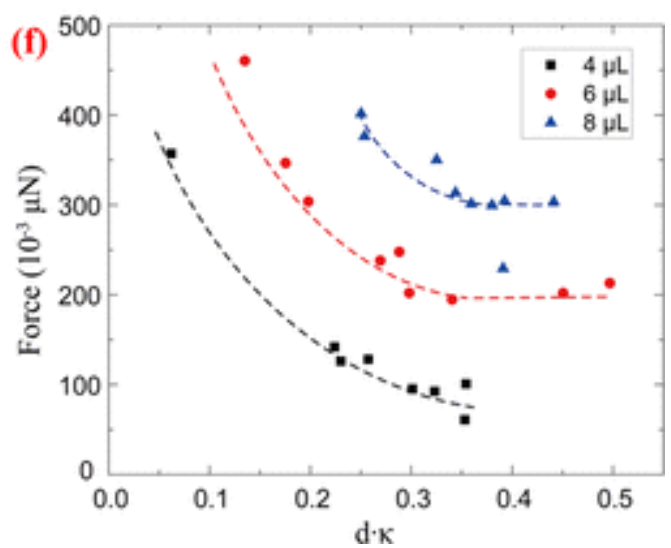

Figure 2. (a) Dependence of the critical voltage VC on the electrical conductivity $\mathrm{k}$ of the liquid. The liquid marbles of volume $5.0 \mu \mathrm{L}$ are aqueous $\mathrm{NaCl}$ droplets with different concentrations ranging from 0.001 to $1 \mathrm{M}$ coated by silica nanoparticles. (b) Dependence of the critical voltage VC on the relative electrical permittivity $\varepsilon$ of the liquid. The liquid marbles of volume $5.0 \mu \mathrm{L}$ are aqueous droplets with different concentrations of glycerol from $0 \%$ to $80 \mathrm{wt} \%$ coated by silica nanoparticles. 
The data point for $\varepsilon \approx 72.3$ is the measured values for DI water ( $\approx \approx 0.05 \mu \mathrm{S} / \mathrm{cm}$ ). (c) Dependence of the critical voltage VC on the $\mathrm{d} / \mathrm{R}$ ratio, for DI water marbles of volume $6 \mu \mathrm{L}$ coated by silica nanoparticles. Inset: schematic showing $d$ is measured as the distance between the centroid of the marble and the symmetry line of the two neighboring electrodes. (d) Schematic illustration of the electric stress fe pulls the interface by compressing and pinning the interface to the substrate over a height $\Delta y$ for a small displacement $\Delta x$ of the marble. The maximum electrostatic energy is released to accelerate the marble when the contact area between the marble and the substrate is fully polarized. (e) Log-log plot of the scaling relationship between the kinetic energy and the electrostatic energy for liquid marbles of volume 4,6 , and $8 \mu \mathrm{L}$ and different initial positions of $d / R$ ratio. The black squares, red circles, and blue triangles represent the data for liquid marbles of volume 4,6 , and $8 \mu \mathrm{L}$, respectively. Here, $K=0.68$ is a dimensionless prefactor; the line has a slope of unity. (f) Plot of force estimation during actuation versus the distance $d$ normalized by the capillary length $\mathrm{k}-1$ for liquid marbles of volume 4,6 , and $8 \mu \mathrm{L}$ and different initial positions of $\mathrm{d} / \mathrm{R}$ ratio.

FIGURE 3.

(a)

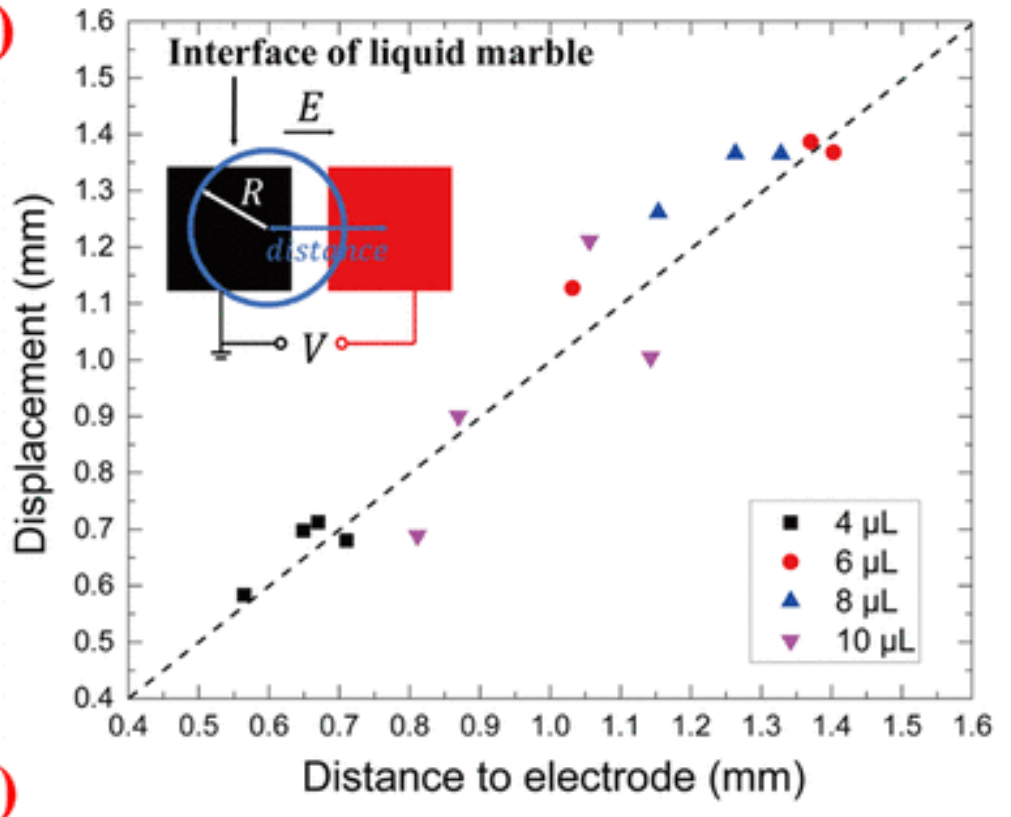

(b)
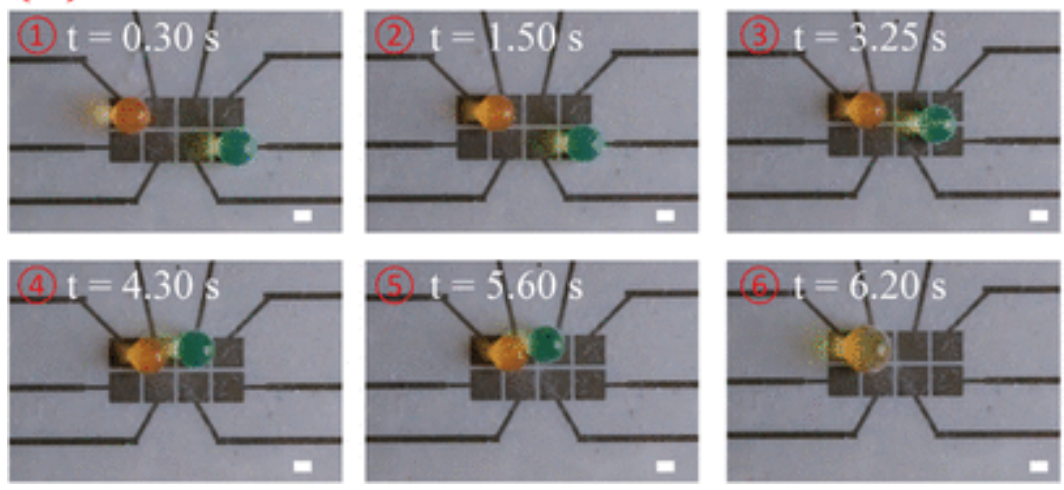

Figure 3. (a) Plot of the total displacement of the marble after actuation versus the distance between the centroid of the marble at its initial position and the symmetry line of the charged electrode. The line has a slope of unity. Inset: schematic showing the measurement of the distance of the marble to the charged electrode. The applied electric potential $V$ falls in the gap between the ground electrode 
in black and the charged electrode in red. (b) Video frame sequence showing that liquid marbles can be individually actuated and coalesced on our device. Two liquid marbles are dyed yellow and green separately. We sequentially apply an electric potential of $800 \mathrm{~V}$ to a series of electrodes and individually actuate the yellow marble and green marble to move, approach each other and finally coalesce into one. Scale bar $=1 \mathrm{~mm}$.

\section{FIGURE 4}

(a)

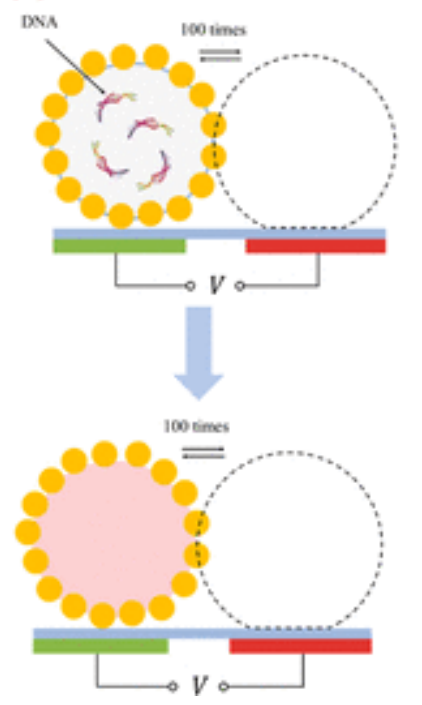

(b)

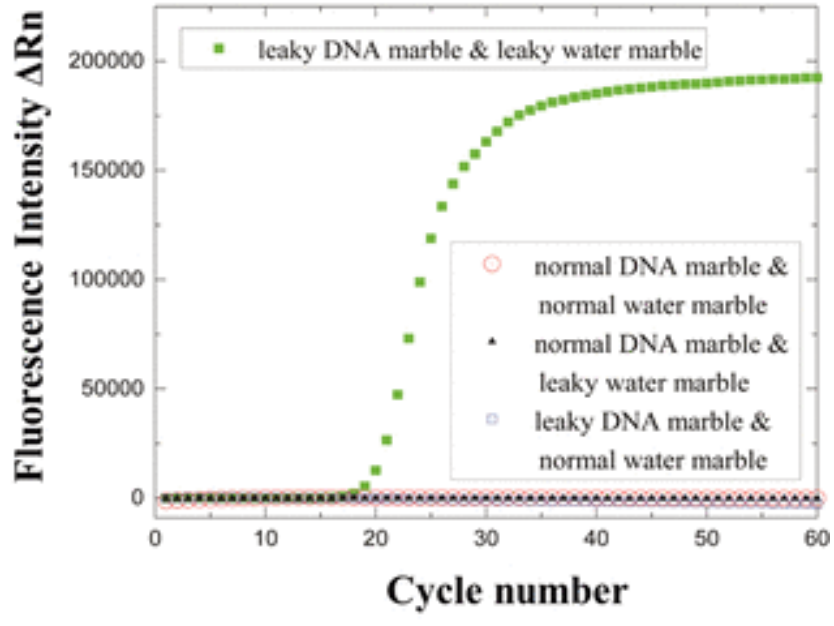

Figure 4. (a) Schematic illustration to confirm the avoidance of cross-contamination between liquid marbles by demonstrating the absence of any trace DNA after a LAMP reaction. Specifically, we actuate a liquid marble containing $1 \times 104$ copies/ $\mu \mathrm{L}$ target DNA to move back-and-forth along a predefined path for 100 times. Then another liquid marble containing DI water is actuated back-andforth along the same path for 100 times. Afterward, the liquid in the second liquid marble is extracted for LAMP reaction to indicate the extent of cross-contamination. (b) Experimental results showing the extent of cross-contamination for different combinations of normal and leaky liquid marbles. Fluorescence intensity $\triangle \mathrm{Rn}$ is the magnitude of normalized fluorescence generated by the FAM/SYBR green reporter with an emission peak of $520 \mathrm{~nm}$ at each cycle during the PCR amplification. When no or only one liquid marble leaks, flat amplification curves are observed indicating that no DNA exists in the second liquid marble. However, an S-shaped curve is found in the LAMP reaction when we actuate two leaky liquid marbles sequentially on the same platform, confirming the cross-contamination between the two leaky liquid marbles. 